\title{
Aviation Emergency Rescue Dispatching Plan Based on Route Optimization Model Considering Random Wind
}

\author{
Hui Yu, Ming Zhang ${ }^{+}$, Zhiyuan Shen and Jue Yu \\ College of Civil Aviation, Nanjing University of Aeronautics and Astronautics, China
}

\begin{abstract}
As an effective means of nature disaster relief, aviation emergency rescue is widely used in many countries. Aviation emergency rescue dispatching plan plays a dominant role that guarantees the efficient implementation of aviation emergency rescue. The conventional dispatching plan without considering random wind factor leads to non-precise quick-responsive scheme and safe issue. In this paper, an aviation emergency rescue framework considering the influence of random wind at flight trajectory is proposed. In this framework, the predicted wind information at disaster area is updated by unscented Kalman filtering (UKF) technology. Then, the motion model of rescue airplane is built by fusing the effect of random wind. An optimization model aiming at maximizing satisfaction index of rescue priority and minimizing total rescue flight distance is formulated. Finally, hill-climbing genetic algorithm is used to solve the mentioned multiple-targets model. A case study with a real earthquake disaster occurring in Sichuan Province is conducted. The results show that the obtained dispatching plan considering the constraints of updated wind speed and direction is more applicable for real operation.
\end{abstract}

Keywords: aviation emergency rescue, dispatching plan, random wind, rescue priority

\section{Introduction}

Aviation emergency rescue dispatching plan plays a dominant role that guarantees the efficient implementation of aviation emergency rescue, which has aroused growing concerns and research interests recently due to the occurrence of several natural disasters (e.g., Iowa floods in Mississippi, US and Wenchuan earthquake in Sichuan Province, China).Every year, more than 500 disasters are estimated to strike our planet, killing around 75,000 people and impacting more than 200 million others [1]. Disasters result in massive demands that often outstrip resources. In order to support rescue operation after disasters, the urgent relief service must be delivered to the affected areas as quickly as possible, i.e., the quick-responsive aviation emergency logistics dispatching is needed for the relief service operations. The process of planning, managing the number of aircraft and the order of airplane on the track is called aviation emergency rescue dispatching plan.

Recently, it is shown that dispatching plan is one of the fundamental problems in emergency logistics management (Davidich and Köster, 2012 [2]; Heliövaara et al., 2012 [3]; Shi et al., 2012 [4]). In order to send the commodities rapidly, the path whose flight distance shortest should be selected. A vast amount of literatures (Sheu, 2007 [5]; Deng and Chan, 2011 [6]; Ozdamar and Pedamallu, 2011 [7]; Hatami Marbini et al., 2013[8]) has been produced up. The conventional dispatching plan pays more attention to demand materials distribution, which leads that multiple real factors affecting quick response of emergency rescue are subordinated. It is well-known that random wind at the range of flight trajectory is the primary factor that causes the deviation between pre-planned flight trajectory and practical flight trajectory in the process of aviation emergency rescue. Furthermore, random wind can not only permute the result of total airplane dispatching plan but also threaten the security of aviation emergency rescue. Thus, to achieve a more

Corresponding author. Tel.: +86-13851656487; fax: +86-25-84891289.

E-mail address: zhangm@nuaa.edu.cn. 
efficient and safer airplane dispatching plan, random wind factor is necessarily considered to aviation emergency rescue model. In this paper, an aviation emergency rescue framework considering the influence of random wind at flight trajectory is proposed.

The paper is organized as follows. In section 2, the updated predicted wind information at the flight trajectory was acquired by UKF fusion technology. Then an airplane motion model with the influence of random wind was built. In section 3, an aviation emergency rescue dispatching plan model with the constraints of rescue priority and random wind was proposed, which was solved by a well-known hill-climbing genetic algorithm. A case study was implemented to verify the feasibility and effectiveness of the model in section 4.Finally, the conclusion was drawn in section 5.

\section{Airplane Motion Model under the Influence of Random Wind}

\subsection{Prediction of random wind based on UKF}

UKF is an algorithm that combines unscented transformation and Kalman filtering [9], and strong stochastic turbulence is the most important character of random wind. It is demonstrated that UKF features the ability to deal with stochastic turbulence. Hence, UKF technology was applied to update the predicted random wind information in this paper. To obtain the more precise random wind information, first, consider international ground exchange station in the rescue airspace as the observation, from which accurate random wind information was acquired, and then, the grid predicted data of random wind, which were stored at NetCDF format, was obtained from World Aero Forecast System. The predicted data was obtained by fusing mentioned weather data based on UKF technology. Each airport is equipped with one or more international ground exchange stations, thus the updated predicted wind information at the flight trajectory between any two distribution centres can be drawn through linear interpolation model.

\subsection{Airplane motion model under the influence of random wind}

In this subsection, an airplane motion model was built. The synthesis processing of random wind speed and true airspeed of airplane was described in Fig. 1.

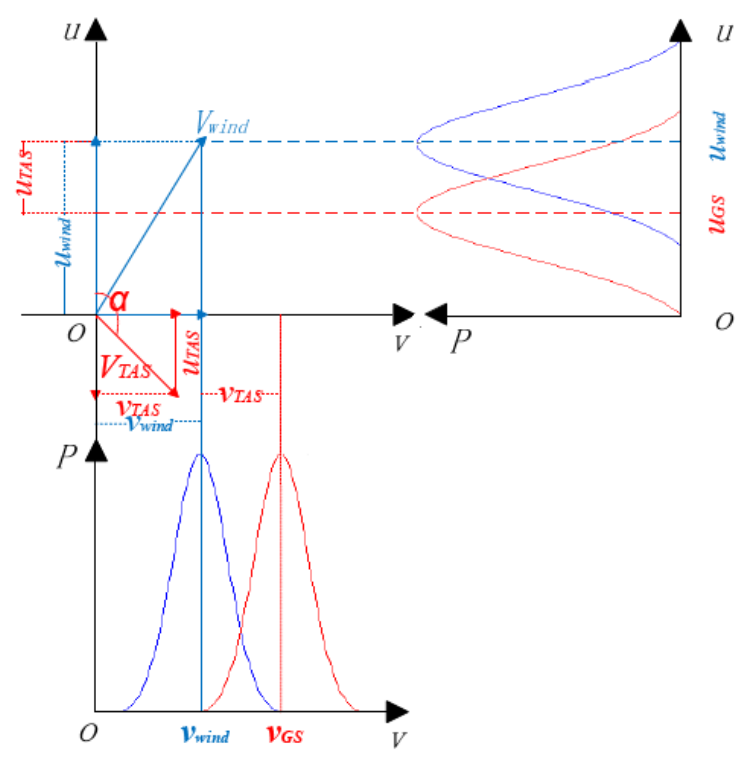

Fig. 1: The normal composite of random wind speed and true airspeed of airplane.

Through analysis the historical random wind data from international ground exchange station, wind speed component $u$ and $v$ were assumed to obey the normal distribution respectively. The blue normal curve represents random wind vector, in which the average speed was $u_{\text {wind }}$ and $v_{\text {wind }}$ in the $u$ and $v$ directions, denoted as $u_{\text {wind }} \sim N\left(u_{\text {wind }}, \sigma_{1}\right)$ and $v_{\text {wind }} \sim N\left(v_{\text {wind }}, \sigma_{2}\right)$. Then the true airspeed was divided into these two directions. The ground speed of airplane, which is the sum velocity of random wind speed and true air speed of airplane, was obtained by vector synthesis principle. Therefore, the red normal curve was defined to describe the ground speed of airplane, in which the average speed was $u_{G S}$ and $v_{G S}$ in the $u$ and $v$ directions, 
denoted as $u_{m k} \sim N\left(u_{G S}, \sigma_{1}\right)$ and $v_{m k} \sim N\left(v_{G S}, \sigma_{2}\right)$.The internal relationships among mentioned parameters satisfied as: $u_{G S}=u_{\text {wind }}+v_{T A S} \sin \alpha$ and $v_{G S}=v_{\text {wind }}+v_{T A S} \cos \alpha$ Consequently, the ground speed of airplane fused with updated predicted random wind velocity also obeys the normal distribution, of which distribution is $V_{m k} \propto \exp \left\{-\left(V_{m k}-u\right)^{2} / 2 \sigma^{2}\right\}$.

\section{Aviation Emergency Rescue Dispatching Plan Model}

In this section, a composite multi-objective optimization model was formulated to deal with the problem of the satisfaction index of rescue priority and the rescue flight distance under the influence of random wind. Each of the given distribution-center group $g$, planned to be served in a given time interval $t$, is associated with the two respective objective functions with the goals of maximizing satisfaction index of rescue priority $F_{g}^{1}(t)$ and minimizing total rescue flight distance $F_{g}^{2}(t) . F_{g}^{1}(t)$ and $F_{g}^{2}(t)$ are given by

$$
\begin{gathered}
\max F_{g}^{1}(t)=\sum_{i=1}^{n_{m k}} W_{i g}\left(n_{m k}+1-r_{m j i}\right) \\
\min F_{g}^{2}(t)=\sum_{m=1}^{M} \sum_{k=1}^{K} \sum_{i=1}^{n_{m k}}\left\{\left(d_{r_{m j(i-1)} r_{m k i}}+d_{r_{m j m_{m k}} r_{m j 0}}\right) \cdot \operatorname{sign}\left(n_{m k}\right)\right\}
\end{gathered}
$$

where $W_{i g}$ represents the rescue priority of each distribution center; $\mathrm{m}$ is defined as the ${ }^{\text {th }}$ retrieval depot, $m=1, \cdots, M ; \mathrm{k}$ is given as the number of airplane in each retrieval depot, $k=1, \cdots, K ; n_{m k}$ denotes the number of distribution center served by airplane $k$ which leaves from retrieval depot $\mathrm{m}$; ${ }^{m j i}$ describes the sequence $i$ of distribution center $i_{g}$ at the flight trajectory $j$ whose starting point is retrieval depot $m$; $d_{r_{m j(i-1)} r_{m j i}}$ represents the distance between two adjacent distribution centers, which are on the same trackjfrom retrieval depot $m$, and $d_{r_{m i m_{k} k} r_{m j} \text {. }}$ represents the distance between retrieval depot $m$ and the latest distribution center on the trackj; $\operatorname{sign}\left(n_{m k}\right)$ is a switch variable, whose value is 0 or 1 .

The built optimization model is constraint with following terms, in which the performance of aircraft and limitation of relief are considered. They are listed on Eqs. (3)-(7)

$$
\begin{aligned}
& d_{r_{m j(i-1)} r_{m k i}}+d_{r_{m j m_{m k} r_{m j 0}}} \cdot \operatorname{sign}\left(n_{m k}\right) \leq\left(f_{m k} / c_{m k}\right) \cdot V_{m k}\left(0 \leq n_{m k} \leq L_{m}\right) \forall m, k \\
& \sum_{m=1}^{M} L_{m}=N \\
& \sum_{k=1}^{K_{m}} n_{m k}=L_{m}(\forall m) \\
& \operatorname{sign}\left(n_{m k}\right)=\left\{\begin{array}{c}
1, n_{m k} \geq 1 \\
0, \text { else }
\end{array}\right. \\
& W_{i g} \in(1,5)
\end{aligned}
$$

in which $f_{m k}$ is defined as the maximum amount of loading fuel for rescue aircraft; $c_{m k}$ is the average fuel consumption rate; $v_{m k}$ denotes the ground speed of airplane fused by random wind speed and true airspeed, which obeys the normal distribution; $L_{m}$ represents the number of distribution center served by retrieval depot $\mathrm{m} ; \mathrm{N}$ describes the sum number of retrieval depots.

In detail, Eq. (3) is specified to ensure that total flight distance of each airplane in the processing of rescue is less than its maximum flight distance; Eq. (4) guarantees that all the distribution center can be served. Furthermore, Eq. (5) indicates that each distribution center can receive only once relief; In Eq. (6), $\operatorname{sign}\left(n_{m k}\right)$ is defined in the case that $n_{m k}$ is greater than or equal to $1, \operatorname{sign}\left(n_{m k}\right)=1$, on the contrary, $\operatorname{sign}\left(n_{m k}\right)=0$; Eq. (7) defines a feasible numerical domain for rescue priority $W_{i g}$.

\section{Simulation and Experiment}

\subsection{Parameter settings}


At this case, the data was based on a real earthquake disaster occurring in Sichuan Province. The performances of dispatched airplane were listed in Tab.1. The random wind information at these areas were reported and download from http://apps.ecmwf.int/datasets/data/interim-full-daily/levtype=sfc/.

TABLE I: THE PERFORMANCE OF DiSPATCHED AIRPLANE

\begin{tabular}{cccc}
\hline Type & $\begin{array}{c}\text { The maximum } \\
\text { amount of fuel }\end{array}$ & $\begin{array}{c}\text { The average fuel } \\
\text { consumption rate }\end{array}$ & $\begin{array}{c}\text { The average } \\
\text { speed }\end{array}$ \\
\hline M171 & $2732 \mathrm{~kg}$ & $0.39 \mathrm{~kg} / \mathrm{kw} \cdot \mathrm{h}$ & $230 \mathrm{~km} / \mathrm{h}$ \\
M8 & $2027 \mathrm{~kg}$ & $0.36 \mathrm{~kg} / \mathrm{kw} \cdot \mathrm{h}$ & $180 \mathrm{~km} / \mathrm{h}$ \\
\hline
\end{tabular}

Generally speaking, in the process of aviation emergency rescue dispatching plan, as shipping point, airport is the first choice. In this paper, two types of shipping point were defined in Tab. 2.

TABLE II: THE ESSENTIAL ATTRIBUTES OF EACH POT

\begin{tabular}{cccc}
\hline No. & Name & Type & Rescue Priority \\
\hline 1 & Chengdu Shuangliu Airport & retrieval depot & null \\
2 & Yibing Airport & retrieval depot & null \\
3 & Aba Hongyuan Airport & distribution center & 5 \\
4 & MianyangNanjiao Airport & distribution center & 5 \\
5 & XichangQingshan Airport & distribution center & 4 \\
6 & GanziKangding Airport & distribution center & 4 \\
7 & Jiuzhaihuanglong Airport & distribution center & 3 \\
8 & DaZhouHeshi Airport & distribution center & 3 \\
9 & Ganzi Aden Daocheng Airport & distribution center & 3 \\
10 & PanzhihuaBao'anying Airport & distribution center & 3 \\
11 & GuangyuanPanlong Airport & distribution center & 2 \\
12 & NanchongGaoping Airport & distribution center & 2 \\
13 & Leshan Tourism Airport & distribution center & 2 \\
14 & LuzhouLantian Airport & distribution center & 1 \\
\hline
\end{tabular}

\subsection{Results and analysis}

A well-known hill-climbing genetic algorithm [10] was used to solve previous mentioned model in Eq. (1)-(7). The result of dispatching plan of airplane was shown in Fig. 2.

The results in Fig. 2 can be explained by Tab. 3 in detail. For Yibing airport, the dotted line shown at the bottom of Fig. 2 is expressed at the last column of Tab. 3, where the order of dispatching plan is Yibing Xichang Qingshan - Panzhihua Bao'anying - Luzhou Lantian - Yibing, and the total route distance is $914.4 \mathrm{~km}$.

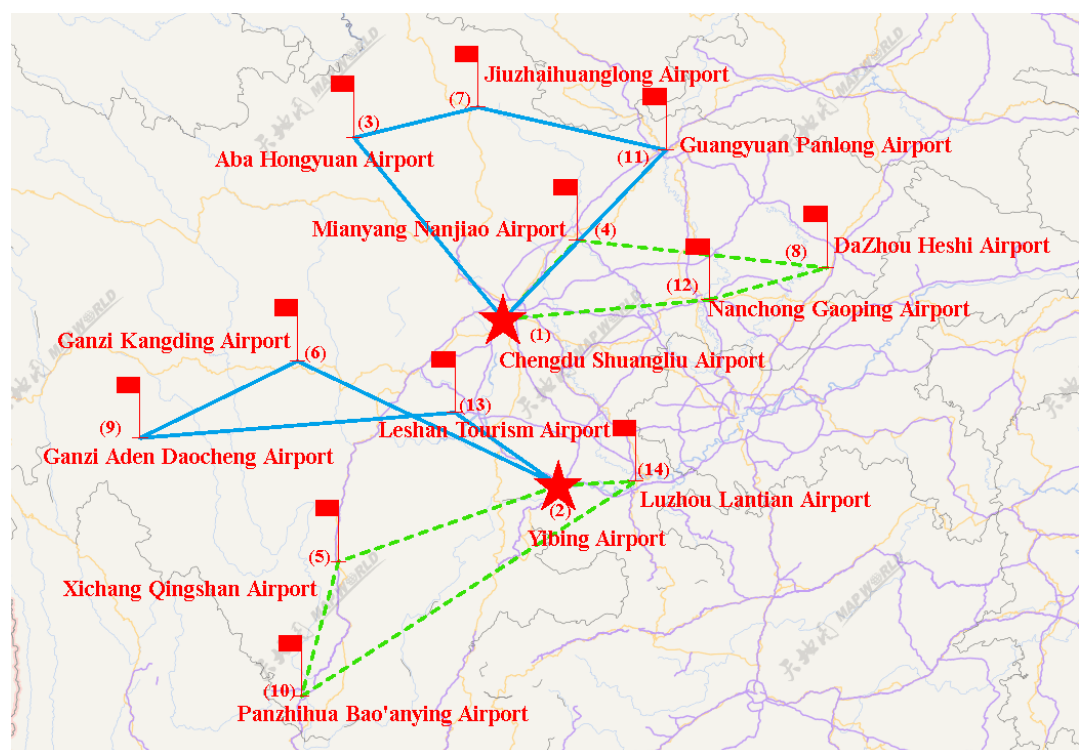

Fig 2: A real operation map of aviation emergency rescue dispatching plan. 
TABLE III: Dispatching Plan OF RESCUE AIRPLANE

\begin{tabular}{ccccc}
\hline & Chengdu Shuangliu Airport (1) & \multicolumn{2}{c}{ Yibing Airport (2) } \\
\hline Group & \multicolumn{2}{c}{347811 13 } & \multicolumn{2}{c}{569101314} \\
Type & M171 & M8 & M171 & M8 \\
Route & $1-3-7-11-1$ & $1-4-8-13-1$ & $2-6-9-13-2$ & $2-5-10-14-2$ \\
Mileage & $849.7 \mathrm{~km}$ & $716.8 \mathrm{~km}$ & $963.9 \mathrm{~km}$ & $914.4 \mathrm{~km}$ \\
Total Mileage & \multicolumn{2}{c}{$1566.5 \mathrm{~km}$} & \multicolumn{2}{c}{$1878.3 \mathrm{~km}$} \\
\hline
\end{tabular}

\section{Conclusion}

In this work, an aviation emergency rescue framework considering the influence of random wind at flight trajectory is proposed. In this framework, the revised predicted wind information at the flight trajectory is updated by UKF technology. With the constraints of updated wind speed and direction, an optimization model aiming at maximizing satisfaction index of rescue priority and minimizing total rescue flight distance is built. Finally, hill-climbing genetic algorithm is used to solve the mentioned multiple targets model. A case study with a real earthquake disaster occurring in Sichuan Province is conducted. The results show that the obtained dispatching plan considering random wind is more applicable for real operation.

\section{Acknowledgements}

The authors would like to thank the financial support by National Natural Science Foundation of China (No.U1233101 and No.71271113) and Fundamental Research Funds for the Central Universities (NS2016062).

\section{References}

[1] Wassenhove LNV. Humanitarian aid logistics: supply chain management in high gear. The Journal of the Operational Research Society 2006;57(5):475-89.

[2] Davidich, M., Köster, G., 2012. Towards automatic and robust adjustment of human behavioral parameters in a pedestrian stream model to measured data. Safety Science 50 (5), 1253-1260.

[3] Heliövaara, S., Kuusinen, J., Rinne, T., Korhonen, T., Ehtamo, H., 2012. Pedestrian behavior and exit selection in evacuation of a corridor: an experimental study.Safety Science 50 (2), 221-227.

[4] Shi, C., Zhong, M., Nong, X., He, L., Shi, J., Feng, G., 2012. Modeling and safety strategy of passenger evacuation in a metro station in China. Safety Science 50 (5), 1319-1332.

[5] Sheu, J., 2007. An emergency logistics distribution approach for quick response to urgent relief demand in disasters. Transportation Research Part E: Logistics and Transportation Review 43 (6), 687-709.

[6] Deng, Y., Chan, F., 2011. A new fuzzy dempster MCDM method and its application in supplier selection. Expert Systems with Applications 38 (8), 9854-9861.

[7] Ozdamar, L., Pedamallu, C., 2011. A comparison of two mathematical models for earthquake relief logistics. International Journal of Logistics Systems and Management 10 (3), 361-373.

[8] Hatami-Marbini, A., Tavana, M., Moradi, M., Kangi, F., 2013. A fuzzy group Electre method for safety and health assessment in hazardous waste recycling facilities. Safety Science 51 (1), 414-426.

[9] Kandepu R, Foss B, Imsland L. Applying the unscented Kalman filter for nonlinear state estimation. Journal of Process Control.2008;18(7): 753-768.

[10] Vo T Q, Kim H S, Lee B R. Propulsive Velocity Optimization of 3-Joint Fish Robot Using Genetic-Hill Climbing Algorithm[J]. Journal of Bionic Engineering, 2009, 6(4):415-429. 